\title{
Succinyl-CoA:3-Ketoacid Coenzyme A Transferase 1, Mitochondrial
}

National Cancer Institute

\section{Source}

National Cancer Institute. Succinyl-CoA:3-Ketoacid Coenzyme A Transferase 1,

Mitochondrial. NCI Thesaurus. Code C158470.

Succinyl-CoA:3-ketoacid coenzyme A transferase 1, mitochondrial (520 aa, $\sim 56 \mathrm{kDa}$ ) is encoded by the human OXCT 1 gene. This protein plays a role in the catabolism of ketone bodies. 